\title{
EI Campus virtual de la FES Zaragoza-UNAM: Innovación en la organización
}

\author{
The virtual campus of FES Zaragoza-UNAM: Innovation in the \\ organization
}

\author{
Alma Xóchitl Herrera Márquez ${ }^{1}$, Juan Luis Soto Espinosa ${ }^{1}$, \\ y Patricia Parra Cervantes ${ }^{1}$
}

\begin{abstract}
${ }^{1}$ Facultad de Estudios Superiores Zaragoza - UNAM, Ciudad de México, México. almaherreramarquez@gmail.com,soej@unam.mx,pparra@unam.mx
\end{abstract}

(Recibido: 15 noviembre 2019; aceptado: 17 febrero 2020)

\begin{abstract}
Resumen. El Campus virtual tiene varios objetivos: fortalecer la formación universitaria mediante el diseño de modelos y prácticas educativas mediadas por tecnologías digitales; crear comunidades de aprendizaje y redes de conocimiento; promover el acceso libre a la información y al conocimiento con responsabilidad social; y brindar servicios de asesoría, capacitación, soporte y desarrollo de recursos digitales a profesores e investigadores en las áreas de formación de la facultad. Se articulan cuatro planes maestros vinculados a cuatro procesos de gestión específicos: "Aprendizaje", "Producción del Conocimiento", "Uso y Distribución Social del Conocimiento" y "Mejora Continua". Los diversos programas han consolidado el uso crítico de las TIC en la formación de los estudiantes y de la formación de cuadros docentes capaces de generar, promover y conducir innovaciones en los procesos educativos, los contenidos, los métodos, las prácticas y los medios dirigidos a mejorar la calidad del aprendizaje en todo tipo de ambiente educativo.

Palabras clave: Campus virtual, TIC, Comunidades de aprendizaje, Redes de conocimiento.

Abstract. The virtual campus aims to strengthen university education through the design of educational models and practices mediated by digital technologies; create learning communities and knowledge networks; free access to information and knowledge with social responsibility; provide consulting services, training, support and development of digital resources services to teachers and researchers in the areas of teacher training. Four Master Plans linked to four specific Management Processes: Learning, Knowledge Production, Use and Social Distribution of Knowledge and Continuous Improvement. Some programs have consolidated the critical use of TIC in the training of students and the training of teaching staff capable of generating, promoting and carrying out innovations in the processes, contents, methods, practices and educational means aimed at improving the quality of the learning in all types of educational environment.
\end{abstract}

Keywords: Virtual campus, ICT, Learning communities, Knowledge networks.

\section{Introducción}

El concepto de Campus universitario alude a un espacio o terreno que delimita las fronteras físicas de la universidad. El campus es considerado como un recinto universitario. Si bien, físicamente, el campus se encuentra integrado por un conjunto de edificios organizados para desarrollar las funciones usuales de las universidades, el concepto campus es mucho más comprehensivo. Como señala Behm Rosas (1969, p.1072

Cite this work as Herrera Márquez A., Soto Espinosa J., Parra Cervantes P. (2020). El Campus virtual de la FES Zaragoza-UNAM: Innovación en la organización. Revista Colombiana de Computación, 21(1), 35-45. https://doi.org/10.29375/25392115.3897 
citado por Fuentes Hernández, 2007, p.121), en el campus se sintetiza una forma específica de interpretar y darle un significado al espacio arquitectónico; refleja la organización de las disciplinas en armonía con el conjunto de edificios, los pasillos que conectan la vida académica, los salones reservados a la realización de un experimento o al dictado de un cátedra, las bibliotecas centradas en la organización de la información con lógicas diversas y flexibles, la diferenciación entre los espacios destinados para la formación universitaria, o la investigación y extensión de la cultura. Todo eso articula el concepto de universidad que se tenga.

El campus forma parte de un contexto, sea una ciudad, una zona rural o el ciberespacio, pero al mismo tiempo arropa a una comunidad interesada en aprender, conocer, generar o difundir conocimiento; tiene una identidad propia que fortalece el sentido de pertenencia a una comunidad académica.

Campus [físico o virtual], es el ámbito [espacio] donde transcurre la vida universitaria, fuente de conocimiento, enseñanza y reflexión disciplinaria e interdisciplinaria y, por tanto, tiene un alcance cualitativo que supera un problema simplemente dimensional [...] su significado cultural, la complejidad de sus funciones educacionales y la escala de la masa estudiantil que se reúne y desplaza en forma colectiva por su interior constituyen la medida determinante de sus espacios. (Fuentes Hernández, 2007, p. 128).

Bajo esta perspectiva, el campus es una ecología o arreglo ambiental - affordance, según Gibson (1979) y citado por Davisson y Leone (2017) - que posibilita la adaptación, predispone la anticipación, favorece la imaginación, moldea el desarrollo de conductas y propicia la acción en un espacio y tiempo determinados. Una affordance está compuesta por recursos o soportes ambientales que son percibidos por los individuos para usarlos y responder a las condiciones y demandas del entorno. En los ecosistemas biológicos, las affordances son procesos que ocurren de manera automática como, por ejemplo, un ambiente pantanoso, que tiene características que favorecen la supervivencia de los insectos acuáticos, pero dificultan el movimiento y supervivencia de mamíferos cuadrúpedos.

Los cambios experimentados en el último siglo son de tal magnitud, velocidad, profundidad y alcance que no sólo han transformado el entorno externo facilitando e innovando la acción humana; sino que también han transformado su mente y cognición (Gere, 2009). De acuerdo con Clark y Chalmers (2011), así como con Sánchez Sordo (2019), con las tecnologías digitales la cognición puede ir más allá de los límites de la piel, ya que no es un proceso necesariamente intracraneal, sino que ocurre también desde los dispositivos (artefactos). Es decir, que la mente integra en el proceso cognoscitivo a las herramientas para poder realizar tareas concretas, siendo entonces los dispositivos o artefactos tecnológicos una especie de extensión o prótesis en la cual la acción cognitiva tiene lugar.

La cultura digital, promovida por los campus virtuales abarca más allá de los sistemas, prácticas, entornos y medios culturales simbólicos (como los directamente relacionados con la información, la comunicación, el conocimiento o la educación) y se extiende prácticamente por todos los ámbitos de la sociedad digital [pues] los rápidos procesos de innovación desencadenados por las TIC digitales han transformado radicalmente, junto con los sistemas, los colectivos y las dinámicas de la información y la comunicación, también las formas de conocimiento e investigación tecno-científica. (Lévy, 2007, p.129).

Davisson y Leone (2017) señalan que en los campus virtuales (entornos digitales) las affordances no son naturales, sino que están diseñadas por los creadores de los sitios, para dirigir al usuario a navegar en entornos virtuales de una forma específica y, por lo tanto, el usuario debe estar preparado para percibirlas y para usarlas. Cada entorno digital es una affordance y cada uno corresponde conductas distintas y procesos de navegación diferenciales que dependen de las características de la interfaz. En su nivel más básico, son las características de la interfaz de un dispositivo y de las posibilidades de acción que experimenta una persona las que predicen la intensidad de su uso. Los usuarios de Internet experimentan las affordances de muchas maneras y responden a ellas en función de sus capacidades, intereses y aptitudes personales. En este nuevo mundo digital, donde la tecnología de ninguna manera puede considerarse una moda pasajera, la nueva información convierte la ideología de una cultura en software cultural, como afirma J. E. Clark (2010).

Budka (2011) plantea que este es un nuevo fenómeno sociocultural en el que en la "web asociativa" se mezclan lo material, lo social y lo simbólico; elementos que luego son procesados con un chip de computadora, de modo que se generan nuevas relaciones y roles entre actores y emergen e interactúan múltiples literacidades y diferentes sistemas semióticos que exigen el dominio en el uso de tecnologías multimedia. Así, es claro que el Campus universitario articule entornos físicos con entornos virtuales que 
provean a los estudiantes múltiples ambientes de aprendizaje y un importante conjunto de herramientas que beneficien el proceso educativo.

Las universidades españolas se encuentran en una fase expansiva de creación y desarrollo de campus o servicios virtuales para la docencia universitaria [...] podemos observar tres modelos organizativos en la oferta universitaria: campus virtual de una universidad presencial, universidad virtual y campus virtual interuniversitario. (Urbina y Salinas, 2014, p.3).

Este mismo fenómeno se observa también en México ${ }^{1}$. Esta tendencia inició desde los años 70, pero ya a finales del siglo XX se consideraba que el campus virtual podía ser cualquier cosa, desde una colección de páginas web que describen cursos hasta un entorno de realidad virtual en 3D para el aprendizaje (Maher, Skow y Cicognani, 1999).

El campus virtual o la universidad virtual se pueden definir como un conjunto de funciones habilitadas por la tecnología que hacen posibles interacciones entre diferentes grupos en la universidad (estudiantes, profesorado, gestión y personal de apoyo) sin la necesidad de coincidir en el tiempo o el espacio. (Rickards, 2000, p.1).

Así, crece la posibilidad de crear campus que sean tanto físicos como virtuales, que busquen multiplicar las fortalezas de los dos entornos.

\section{Estudio de caso: la Facultad de Estudios Superiores Zaragoza}

En México, la educación superior se encuentra en el centro de profundas transformaciones y de complejas redefiniciones. La modalidad abierta y a distancia, más allá de permitir sortear las barreras del tiempo y el espacio, se convierte en una oportunidad de formación flexible y continua para avanzar en el aprendizaje a lo largo de toda la vida. Por ello, uno de los principales desafíos que enfrenta la Universidad Nacional Autónoma de México (UNAM) es extender del uso de las nuevas tecnologías de la información y las telecomunicaciones, de tal forma que se mejore la calidad de los procesos educativos a partir de modelos cada vez más orientados hacia la autoenseñanza y a las formas no presenciales de educación.

El avance de proyectos que la UNAM ha impulsado se observan con: a) el desarrollo de tecnologías digitales que articulan sistemas de aprendizaje alternativos; b) la creación de espacios virtuales que han transformado los espacios escolares convencionales (bibliotecas y laboratorios, sobre todo); c) el incremento de redes y comunidades de aprendizaje y práctica (académicas y no académicas); d) la gestión de información multimodal (sonidos, imágenes, textos, hipervínculos) que nos sitúan en un nuevo contexto del conocimiento; y e) la creación del Sistema Universidad Abierta y Educación a Distancia ${ }^{2}$ que ha impulsado la articulación orgánica de las TIC en todas las modalidades y niveles educativos, incluidos el desarrollo curricular, la formación docente, la administración y gestión institucional, y el fortalecimiento del concepto de aprendizaje permanente.

Por otra parte, la madurez institucional de la Facultad de Estudios Superiores Zaragoza (FES Zaragoza) en el campo de las Tecnologías de Información y Comunicación culmina con la creación del Centro de Tecnologías para el Aprendizaje (CETA) cuyo propósito es articular todos los esfuerzos institucionales orientados a generar nuevos acervos universitarios digitales que mejoren la calidad del aprendizaje en todos sus niveles, programas y funciones sustantivas. En el CETA se anida el Campus Virtual de la FES Zaragoza. El campus virtual de la FES Zaragoza constituye, la posibilidad de convergencia académica y cultural en un escenario digital que favorece el encuentro cotidiano de las comunidades de aprendizaje en un ethos de innovación educativa permanente; por ello se conceptualiza como un espacio que tiene alto contenido social, cultural y simbólico para la comunidad.

El Centro de Tecnologías para el Aprendizaje (CETA), ubicado en el Campo I de la Facultad, pretende conformar y consolidar redes y comunidades de aprendizaje que puedan: formar recursos humanos, desarrollar recursos digitales, crear nuevos entornos pedagógicos que fortalezcan las modalidades

\footnotetext{
${ }^{1}$ Esto es esperable porque de acuerdo con el Instituto Nacional de Estadística, Geografía e Informática (2019) el número de usuarios, mayores de seis años, de Internet en México es de 74,3 millones, que representan el $65.8 \%$ de la población en ese rango de edad y con una diferencia entre género del $3 \%$. Del total de la población usuaria de Internet de seis años o más, el grupo de entre 25 y 34 años es el que registra la mayor proporción, siendo las mujeres en este rango de edad el $10.4 \%$ y los hombres $9.8 \%$. Cabe destacar que el segundo grupo en el uso de Internet es el de 18 a 24 años, (8.6\% hombres y $8.9 \%$ mujeres), por lo que es un verdadero desafío innovar los modelos educativos con otras perspectivas.

2“"El Sistema Universidad Abierta y Educación a Distancia de la UNAM está destinado a extender la educación media superior y superior hacia grandes sectores de la población, por medio de métodos teórico-prácticos de transmisión y evaluación de conocimientos y de la creación de grupos de aprendizaje que trabajan dentro o fuera de los planteles universitarios e impulsar la integración de las tecnologías de la información y comunicación a los procesos educativos. El Sistema es de libre opción para las entidades académicas de la UNAM, así como para los estudiantes. Se exigirán los mismos requisitos que existan en la UNAM para el sistemb opción para las entidades académicas de la UNAM, así como paras los estianis. Se exigirán los mismos requisitos que existan en la UNAM para el señaladas en los artículos $8^{\circ}$ y $9^{\circ}$ del Estatuto General de la UNAM" (Universidad Nacional Autónoma de México, 2009, Cap 1).
} 
educativas presencial, abierta a distancia, llevar a cabo proyectos de investigación y desarrollo tecnológico dirigidos a favorecer la calidad del aprendizaje; ofrecer alternativas que mejoren los índices de aprobación en los módulos o asignaturas de alta reprobación; y optimizar los recursos materiales y la infraestructura institucional.

El campus nace con la convicción de que uno de los pilares más sólidos de la innovación educativa en el siglo XXI son las tecnologías de información y comunicación, debido a su enorme potencial para articular sistemas de signos (lenguaje oral, lenguaje escrito, imágenes, movimiento, símbolos matemáticos, musicalizaciones, entre otros) y para representar y trasmitir información (cantidad, velocidad, accesibilidad, distancia). Las tecnologías digitales están modificando los escenarios educativos, sus actores, formatos, modalidades de organización en el tiempo, en el espacio y los recursos didácticos, y están favoreciendo, a su vez, la emergencia de modelos híbridos de enseñanza y aprendizaje que agregan un alto valor formativo a la experiencia cara a cara. De hecho, el aprendizaje en el siglo XXI está cambiando con la introducción de las tecnologías y con la apropiación de capacidades para acceder a muchas fuentes de información.

El Campus virtual de la FES Zaragoza materializa el proceso de transformación que vive la Facultad hacia una cultura digital que transita del consumo y adquisición del conocimiento a nuevos enfoques y modelos de aprendizaje. "Cultura digital que se gesta en el ciberespacio, asumido como un espacio de conocimiento, con potencial cultural, social y político de intercambio, de inteligencia colectiva y conectiva, de interacción subjetiva (individual y colectiva) para la creación y negociación de visiones y significados" (Rueda, 2006, p.38).

Por ende, no aspira a convertirse en un repositorio de recursos digitales, ni a constituirse en una apología de la tecnología. Su propósito central es innovar, dotar de un nuevo significado el proceso formativo ampliando sus fronteras a nuevos códigos y narrativas derivadas de la cultura digital. Las comunidades de aprendizaje que operen en el CETA se organizan de acuerdo con los siguientes principios:

- Cooperación, que implica trabajar en función de objetivos comunes.

- Interdependencia grupal, que comprende la mutua dependencia con respecto a los logros o a los fracasos. Por tal motivo cada integrante toma conciencia de la necesidad de aportar y contar con aportes positivos hacia la tarea común.

- Responsabilidad individual, que significa la toma particular de compromisos de cada participante de forma distribuida con respecto a la tarea común.

- Solidaridad, asumida como la cohesión o la unión entre las diversas partes. Es el reconocimiento del otro.

- Compromiso mutuo, que se expresa en el hecho de que cada miembro comparte su propio conocimiento y recibe el de otros.

La misión de estas comunidades de aprendizaje es innovar la formación universitaria mediante el diseño de modelos y prácticas educativas mediadas por tecnologías digitales. Para esto hay que crearlas y fortalecerlas, al igual que crear y fortalecer redes de conocimiento; también hay que integrar a los estudiantes en dichas comunidades para que estas fortalezcan la formación teórico-metodológica; promover el acceso libre a la información y al conocimiento actuando con responsabilidad social ante las demandas del aprendizaje; brindar servicios de asesoría, capacitación, soporte y desarrollo de recursos digitales a profesores e investigadores en las áreas de ingeniería química, químico biológicas, de la salud y del comportamiento, a fin de mejorar la calidad del aprendizaje de estudiantes de licenciatura y posgrado. Por último, hay que promover la articulación de múltiples ambientes de aprendizaje como respuesta a los desafíos de las tecnologías de información y comunicación. Todo esto tiene proyección institucional, regional, nacional e internacional y al mismo tiempo enriquece su identidad y pertenencia a la UNAM.

\section{Metodología}

Para cumplir su misión, el Campus virtual de la FES Zaragoza articula cuatro planes maestros vinculados a cuatro procesos de gestión específicos:

Plan Maestro de Gestión de Aprendizaje: es el proceso por el que las instancias que intervienen en el desarrollo académico de la institución promueven y contribuyen al logro de una mayor calidad y eficiencia en la formación universitaria, como la formación integral del educando, el aprendizaje autónomo, la flexibilidad o el perfil del profesorado para todos los niveles (pregrado y posgrado). 
Plan Maestro de Gestión de la Producción y Generación del Conocimiento: entendido como el proceso por el que las investigaciones básicas y la aplicadas son vertientes de una de sus funciones esenciales. Por medio de la investigación, no solo se contribuye al desarrollo del saber, sino que se asume el compromiso de generar conocimiento.

Plan Maestro de Gestión del Uso y Distribución del Conocimiento: los programas de educación continua, difusión cultural y de extensión de los servicios a la comunidad tienen un papel importante al ser concebidos y desarrollados como parte de las actividades de una mayor vinculación con la sociedad. Esto con el objetivo de brindar lo que como universidad proyecte.

Plan Maestro de Gestión de Mejora Continua: apoya a las funciones sustantivas de docencia, investigación, extensión y difusión de la cultura. Por lo tanto, comprende los siguientes ámbitos: escolar, gobernanza, financiamiento, desarrollo y cultura institucional.

A su vez, los planes maestros integran programas específicos que consolidan el uso crítico de las TIC en las funciones respectivas de la FES Zaragoza.

Plan maestro: Gestión del Aprendizaje

a. Programa: Talento Docente

b. Programa: Desarrollo de Recursos Digitales para el mejoramiento del Aprendizaje

c. Programa: Innovación Educativa y Educación en Línea (oferta profesional, modelo educativo y modelo pedagógico).

Plan maestro: Gestión en la Producción de Conocimiento

a. Programa: Investigación y Desarrollo Tecnológico

Plan Maestro: Gestión del Uso y distribución Social del Conocimiento

a. Programa: Educación Continua y Capacitación para la vida y el trabajo el trabajo

Plan maestro: Gestión de Mejora Continua

a. Programa: Agenda Digital

b. Programa: Biblioteca Digital

c. Programa: Mejora Continua

Los planes maestros, a su vez, relacionan las siguientes dimensiones:

Fortalecimiento de la formación integral de los estudiantes: su objetivo es diseñar modelos educativos, pedagógicos y curriculares que aprovechen las tecnologías digitales en la formación integral. Esta dimensión abarca objetivos y estrategias relacionadas con la evaluación y actualización permanente de planes de estudio, creación de una nueva oferta educativa exclusiva de las modalidades abierta y a distancia, el incremento de la eficiencia terminal, la disminución de los índices de reprobación y el uso crítico, extenso e intenso de las TIC.

Fortalecimiento de la planta académica (asesores y tutores): los objetivos y estrategias que articulan esta dimensión se dirigen a la conformación de un nuevo perfil de docente caracterizado por el ethos de la innovación permanente.

Fortalecimiento de la innovación tecnológica y la investigación educativa: esta dimensión se dirige al desarrollo de nuevos modelos educativos basados en tecnologías digitales. En este sentido, los planes maestros de Gestión del Aprendizaje y de Producción de Conocimiento deben ser evaluados por su contribución en el mejoramiento de la calidad del aprendizaje y en la elevación del índice de retención.

Impulso a la extensión universitaria: su objetivo es, primordialmente, promover la utilidad social del conocimiento en la vida productiva y en el desarrollo humano sostenible. Contribuye a la mejora de la calidad de vida de la población.

Financiamiento: esta dimensión conduce a estimular la aprobación de proyectos que realicen aportes de financiamientos extraordinarios para el desarrollo de tecnologías digitales para el aprendizaje.

Infraestructura: esta dimensión orienta recursos y esfuerzos compartidos para mejorar sustancialmente las instalaciones, fortalecer la infraestructura y generar estrategias que favorezcan el desarrollo de sistemas inteligentes, ahorro de energía eléctrica, eliminación de desechos y otras medidas sustentables. Esta dimensión coadyuva al mejoramiento de los procesos de aprendizaje e investigación y facilita el desarrollo de redes del conocimiento con nuevas tecnologías, adquisición de acervos y formación de personal especializado, entre otros. 


\section{Resultados}

En el Proceso de Gestión del Aprendizaje es preciso señalar que uno de los propósitos del Campus fue formar cuadros docentes capaces de generar, promover y conducir innovaciones de distinto espectro en los procesos educativos, los contenidos, los métodos, las prácticas y los medios dirigidos a mejorar la calidad del aprendizaje en cualquier ambiente educativo. Para ello, se promovió la apropiación de competencias que desarrollen la capacidad crítica de los docentes para elegir y generar tecnologías, partiendo de la compleja naturaleza del aprendizaje adulto, de las características del modelo didáctico de la educación en línea y de la comprensión cognitiva de los roles que corresponden a los mediadores, las mediaciones y los medios.

El diplomado Práctica Innovadora Educativa con Tecnología Digital Integrada se impartió en dos emisiones en las que se inscribieron 81 académicos. Sin embargo, solo lo concluyeron 55 con la elaboración de un blog educativo de su práctica docente. Este Diplomado fue impartido en colaboración con la Universidad Abierta y a Distancia de México.

Se impartieron 59 cursos y se atendieron a 1,156 asistentes. Las temáticas que se abordaron fueron, entre otras: Apps educativas; búsqueda y validación de la información en la web; búsqueda y recuperación de información por medios digitales; creación de blogs educativos; creación de libros electrónicos con apoyo del programa iBooks Author; creación de un blog educativo en WordPress; curso Aspen One Hysis; curso de Apps para la enseñanza; curso de Google Apps; desarrollo de páginas web educativas; desarrollo de tutoriales interactivos; desarrollo de hojas de cálculo colaborativas como contribución a la evaluación del aprendizaje; escenarios educativos en el contexto digital; diseño, planeación curricular y desarrollo de un Ambiente Virtual de Aprendizaje (AVA); el docente y el alumno en la era digital; elaboración de carteles de investigación dinámicos con Glogster; elaboración de página web educativa; evaluación del proceso de enseñanza y aprendizaje con el empleo de recursos digitales; Excel básico; Excel intermedio para la docencia; fotografía digital; fotografía digital con apps; Google apps para la educación; integración de un plan de clase con el empleo de recursos digitales 2016; manejo del pizarrón electrónico; modelos 3D con VRML y producción de podcast educativo.

Los proyectos de docencia se registran en un sistema especial. Los productos esperados de los proyectos de docencia abarcan fundamentalmente tres categorías:

a. Innovaciones en la práctica educativa.

b. Desarrollo de recursos de aprendizaje con tecnología digital.

c. Elaboración de proyectos de investigación que generen prácticas educativas innovadoras con tecnologías educativas.

Se diseñaron 156 aulas virtuales distribuidas entre las diferentes carreras e instancias de la FES Zaragoza, dando un total de 156 cursos de Ciencias Químico - Biológicas, Salud en el Trabajo, Lenguas Extranjeras, Psicología y Biología.

En el Proceso de Gestión de Generación y Producción del Conocimiento se impulsó el desarrollo de investigaciones dirigidas a integrar todos los esfuerzos de formación de recursos humanos y a aprovechar óptimamente los recursos materiales y la infraestructura. La suma de apoyos de diversos proyectos permitió cohesionar a profesores y especialistas que desarrollaron 645 recursos didácticos digitales para el aprendizaje. Las áreas que han desarrollado recursos digitales son: el Centro de Tecnologías para el Aprendizaje, el Departamento de Actividades Culturales, el Departamento de Certificación Académica, el Departamento de Evaluación Educativa, el Departamento de Lenguas Extranjeras, la Unidad de Formación Integral y la Secretaría de Integración, Promoción y Desarrollo Académico. Estos recursos corresponden a una amplia gama de temáticas, asignaturas y actividades culturales y de educación continua.

La estrategia que se siguió en este Proceso de Gestión agrupó los esfuerzos individuales en tres vertientes:

Vertiente 1: formación de competencias digitales en la planta de profesores de la FES Zaragoza.

Vertiente 2: desarrollo de recursos digitales en temáticas curriculares específicas.

Vertiente 3: desarrollo de tecnologías para el mejoramiento del aprendizaje.

Por otro lado, los cuatro ejes que articularon el desarrollo de la investigación fueron:

1. Recursos digitales para el mejoramiento del aprendizaje.

2. Materiales interactivos en la enseñanza de las Ciencias de la Salud.

3. Digitalización de recursos para el aprendizaje en asignaturas con altos índices de reprobación.

4. Formación integral permanente

La investigación realizada en este Proceso de Gestión se cimentó en el hecho de que el expansivo desarrollo de las tecnologías digitales ha propiciado el surgimiento de una nueva ecología del aprendizaje 
caracterizada por las iniciativas que favorecen el código abierto y el acceso libre a la información, así como la transformación de las prácticas educativas que se enriquecen con el empleo de recursos digitales, el carácter distribuido del conocimiento y la colaboración como condición indispensable para comprender y enfrentar el mundo de hoy.

El Proyecto PAPIIT Rasgos Psico-Educativos que determinan el perfil ideal del estudiante a distancia (IN403315), que se desarrolló en el CETA, permitió generar el Sistema de Perfiles Educativos Recurrentes para Sistemas de Enseñanza Optimizada (PERSEO): Evaluación del Perfil Cognitivo del Estudiante Universitario, cuyo objetivo es que el propio alumno sea consciente de lo que piensa y de cómo piensa para que, a largo plazo, él mismo pueda analizarlo y modificarlo de manera autónoma según sus necesidades. Esto con el propósito de que cualquier estudiante sepa cómo aprende y por qué aprende de determinada manera. Este sistema (PERSEO) se aplicó con 2,901 estudiantes de licenciatura del Sistema de Universidad Abierta y Educación a Distancia (SUAyED) perteneciente a la UNAM. El proceso se realizó en el marco del proceso de selección de la generación 2016-1.

En la etapa actual del proyecto se pretenden desarrollar recursos digitales de aprendizaje, individualizados, flexibles y adaptables, para el apoyo de los procesos de enseñanza-aprendizaje. Con estos recursos se propiciará la apropiación de las competencias individuales y colaborativas de estudiantes y docentes que contribuyan al mejoramiento de la calidad del aprendizaje. Lo novedoso de los recursos digitales que se desarrollarán es que partirán de la evaluación del perfil cognitivo de los estudiantes. Dicho perfil asume que aprender consiste en aplicar todos los talentos de un ser humano para adquirir, comprender y organizar el conocimiento. Aprender implica situarse adecuadamente ante los contenidos, interpretarlos, asimilarlos, retenerlos y aplicarlos en la vida cotidiana. No hay duda acerca de que el aprendizaje tiene un efecto crítico en la forma de entender este complejo e incierto mundo, y de relacionarse con él. La evaluación del perfil considera que todos tenemos un enorme potencial de aprendizaje, aunque nuestros talentos se expresen de diferente forma. Ello se ha corroborado con la sofisticada tecnología utilizada para los estudios de resonancia magnética que registran el interior del cerebro.

Para el desarrollo de las diversas acciones programadas se conformaron grupos de trabajo en la formación de recursos humanos y el uso académico de las tecnologías digitales así como la producción de recursos digitales para apoyar el aprendizaje de las licenciaturas en las áreas químico biológicas, de la salud, del comportamiento, ingenierías y lenguas extranjeras.

La Gestión del Uso y Distribución Social del Aprendizaje se concretó con procesos de la alfabetización digital dirigidos a la capacitación de los trabajadores de base de la FES Zaragoza. Los cursos se relacionan con el manejo de procesadores de texto (Word) y con la introducción al cómputo, principalmente. También se llevó a cabo el Primer Seminario especializado en fuerzas tecnológicas que modelarán el futuro de la educación superior y tres talleres de Programación Creativa en niños, adolescentes y jóvenes.

Por otro lado, se realizó la apertura y administración del canal de Youtube del CETA (https://www. youtube.com/channel/UC17RcmJ-BDVDltSK11nXrxw). Este canal cuenta hasta el momento con los siguientes videos:

1. Uso de Mindmup en Moodle 2.5: 59 visualizaciones.

2. Cómo conectar el pizarrón interactivo (PDI): 50 visualizaciones.

3. Virtualizando la arquitectura del CETA: 21 visualizaciones.

4. Impresión $3 D$ de la espiga FES, Zaragoza, UNAM: 18 visualizaciones.

5. Recorrido Virtual Centro de Tecnologías para el Aprendizaje: 73 visualizaciones.

6. Formaldehído a partir de Metanol simulación con Aspen ONE HYSYS: 434 visualizaciones.

7. Estireno a partir de la deshidrogenación del Etilbenceno simulación con Aspen ONE HYSYS: 242 visualizaciones.

8. Tutorial Introducción al manejo de Aspen One Hysys: 421 visualizaciones.

9. Encuadre y presentación del Curso Taller de Aspen ONE Hysys: 73 visualizaciones.

10. Demostración del manejo del pizarrón interactivo en el área de Lenguas Extranjeras: 89 visualizaciones.

11. Video educativo: 347 visualizaciones.

12. Movimiento CineticomolecularBis: 20 visualizaciones.

13. Aprendizaje demostrativo (11 videos).

14. Gestión de aprendizaje (1 video).

15. Contenido curricular (9 videos). 
Finalmente, el Proceso de Gestión de Mejora Continua dio como resultado:

- Sistema de Gestión de Calidad del Campus virtual, avance 25\%.

- Manual de Calidad con base en los criterios de la Norma ISO 9001-2015, con miras a la certificación.

Para automatizar todos los procesos de registro y seguimiento de procesos y con ello poner en marcha una política de ahorro de papel, con el desarrollo de diversos Sistemas:

- Elaboración de base de datos de registro de proyectos.

- Elaboración de base de datos, actividades del centro, cursos, asistentes, alumnos y profesores.

- Elaboración de base de datos actividades de aulas virtuales.

- Listado de recursos digitales elaborados en el área de psicopedagogía.

- Elaboración de formato de llenado en línea de los proyectos con los que se agiliza el registro y se puede revisar, analizar y elaborar la constancia o documento de registro para el docente.

- Elaboración de control de acceso.

- Elaboración de control de espacios físicos.

- Sistema de registro de proyectos de docencia.

- Sistema de PERSEO.

- Elaboración de una base de datos para llevar acabo el seguimiento de los proyectos que se han registrado, así como de los recursos digitales que se han elaborado.

- Seguimiento de proyectos.

- Rúbricas de Evaluación de Recursos Digitales.

- Inscripción a Talleres Campus III Tlaxcala.

- Evaluación de programas de estudio analíticos (Carrera Cirujano Dentista).

- Sistema de registro de cursos y talleres.

- Creación del canal de Youtube.

- Creación cuenta de Twitter.

- Recorrido virtual.

- Diseño y desarrollo del Sistema distribuido 6th International Congress of the International Commission on Occupational Health - Work Organization and Psychosocial Factors (ICOH-WOPS) Scientific Committee- 2017.

- Diseño y desarrollo el sistema distribuido Red de posgrados ene salud en el trabajo.

El Campus provee servicio y mantenimiento a los equipos del centro en cada una de las salas y espacios multimedia.

\section{Conclusiones}

Para operar con eficiencia, los Campus virtuales requieren tecnologías digitales que favorezcan el diseño y desarrollo de nuevos modelos comunicacionales, procesos de aprendizaje y autoaprendizaje, diseños de estrategias de evaluación, habilidades complejas de pensamiento y de competencias informáticas, modelos educativos flexibles, modelos pedagógicos mediados por TIC, itinerarios personales de aprendizaje, procesos de socialización, congruencia en el diseño de contenidos y materiales con el medio elegido, mecanismos de control y distribución de los contenidos educativos, y procesos de centralización y descentralización de aprendizajes, entre otros.

En los últimos años la presencia de Campus virtuales ha apuntalado seis tendencias de fuerte impacto (García et al., 2010, p. 4):

1. El conocimiento se descentraliza [en todos sus procesos] en producción, distribución y reutilización. [...] Las tecnologías facilitan los procesos de producción y difusión de contenido en múltiples formatos, reproduciendo hasta el infinito la cantidad de recursos explotables en línea.

2. La tecnología sigue afectando profundamente a nuestra forma de trabajar, colaborar, comunicarnos y seguir avanzando. [...] Sin embargo, la rapidez con que evoluciona la tecnología hace que nuevas brechas de acceso (Internet, móvil, banda ancha) y nuevas brechas competenciales (alfabetización digital e informacional) vayan apareciendo antes de cerrarse las anteriores, dando lugar a nuevos escenarios potenciales de desigualdad.

3. La tecnología no solo es un medio para capacitar a los estudiantes, sino que se convierte en un método de comunicación y de relación, así como una parte ubicua y trasparente de su vida. [...] La comunicación entre todos los actores de la educación se ha vuelto más abierta, multidisciplinaria, multisensorial y se va integrando poco a poco a nuestras actividades. 
4. Los docentes y muchas instituciones en las que trabajan, van perdiendo paulatinamente sus recelos hacia las tecnologías, desapareciendo paulatinamente la distinción entre "fuera de línea" y "en línea".

5. Nuestra forma de pensar acerca de los entornos de aprendizaje está cambiando. Hoy día [...] los espacios donde aprenden los estudiantes son cada vez más comunitarios e interdisciplinarios y están apoyados por tecnologías asociadas a la comunicación y a la colaboración virtual. Los espacios se transforman para hibridar lo presencial con lo virtual, difuminándose las fronteras entre ambos mundos, que son vividos por los estudiantes como uno solo.

6. Las tecnologías que usamos se basan cada vez más en estructuras en nube, y nuestra idea de apoyo a las tecnologías de información tiende a descentralizarse. [...] No importa dónde almacenemos nuestro trabajo; lo que importa es que nuestra información sea accesible independientemente de dónde estemos o del dispositivo que hayamos elegido.

Los Campus virtuales favorecen la creación de redes sociales y propician que los universitarios colaboren y realicen investigaciones conjuntas. De hecho, ofrecen al ámbito universitario nuevos espacios de formación, complementarios a la enseñanza presencial, lo que la refuerza. Por otra parte, el entusiasmo que el uso crítico de las TIC ha ocasionado es de tal magnitud que el movimiento en pro de la gratuidad de los recursos educativos ha tenido un gran impulso, facilitando el libre acceso a cursos, planes de estudio y enfoques pedagógicos que no existen localmente.

Las modalidades híbridas pueden llegar a constituir la alternativa estratégica del siglo XXI, dadas las distancias geográficas de un país como México, sus condiciones de vida y la heterogeneidad del nivel educativo $^{3}$. Sin embargo, su efectividad depende de que se superen los factores limitantes que plantean el contexto social particular, la limitada conectividad y los obstáculos derivados de la dinámica institucional. Particularmente en México, los diversos esfuerzos que se han realizado han dado como resultado:

- Una creciente percepción favorable de los docentes acerca de que el Internet promueve el uso de diversas fuentes de información para el desarrollo de sus clases y mejora el proceso de enseñanza mediante la innovación de las prácticas educativas.

- Se motiva más a los alumnos.

- Una mayor articulación con las actividades curriculares.

- Se aprovechan plenamente las tecnologías de la información y la comunicación con fines educativos.

Sin duda, el uso crítico de las tecnologías de información y comunicación aplicadas a la educación constituye una de las más promisorias estrategias para mejorar la cobertura y elevar la calidad de la educación en México. Sin embargo, se requiere comprender la dinámica de las actuales innovaciones. Del mismo modo, estos nuevos entornos de aprendizaje (Campus virtuales) impulsan el diseño de una nueva agenda de trabajo que va desde la conceptualización de las nuevas figuras académicas y de los procesos de aprendizaje emergentes hasta el diseño de innovadoras prácticas educativas y el desarrollo de plataformas tecnológicas más eficientes.

Las tecnologías digitales para el aprendizaje innovan, tanto en la educación presencial, como en las modalidades no presenciales y sostienen el diseño de estrategias de formación que favorecen la educación a lo largo de la vida, impulsan el crecimiento económico y estimulan el desarrollo social. También potencian nuevas y apasionantes posibilidades de estudio e investigación que ya no limitan las fronteras nacionales, pero esto exige infraestructura de educación a distancia a su disposición, además de asesoramiento y asistencia para la creación de las estructuras innovadoras, contenidos pedagógicos compartidos y medios técnicos y humanos para facilitar la preparación de contenidos pedagógicos en la red.

Pese a todo esto, existe un desfase entre la potencialidad de las TIC incorporadas en las aulas y la escasa renovación de los procesos pedagógicos (Meso Ayerdi et al., 2011). Al respecto, Touriñán López et al. (2003, p.61 citado por Marín Díaz y Romero López, 2009) plantean cinco problemas que dificultan la integración de las TIC como herramientas de trabajo diario: (1) la limitación en el acceso a las tecnologías, (2) la rapidez de los avances tecnológicos, (3) la ausencia de una efectiva coordinación de actuaciones en favor de la receptividad de las TIC, (4) la escasa inversión en investigación pedagógica para hacer frente de manera segura a los retos del aprendizaje en la nueva situación, y (5) la necesidad de una planificación efectiva desde la administración educativa respecto de la accesibilidad, receptividad y flexibilidad en la nueva demanda de la educación.

${ }^{3}$ Las estadísticas en la región en relación con la Educación a Distancia muestran un consistente crecimiento de la matrícula. Al respecto, Lupion Torres y Rama (2010) señalan que a partir de la proyección de diversos estudios en algunos países y de los datos en otros, en 2005 había aproximadamente 984 mil estudiantes inscritos en modalidades híbridas, lo que representaba $6.4 \%$ de la matrícula de educación superior de América Latina y el Caribe. 
La apropiación de la cultura digital en educación superior implica el desarrollo de un enfoque pedagógico que promueva el uso crítico de las tecnologías de información y comunicación en el proceso de aprendizaje a partir del conocimiento profundo de los contenidos académicos, el dominio de las competencias pedagógicas, y el manejo y la aplicación de las herramientas digitales. El desafío del presente siglo no tiene que ver con usar o no las tecnologías digitales; muchos ambientes de aprendizaje convencionales tienen poca tecnología o prácticamente no la usan con fines educativos y alcanzan excelentes resultados en la calidad. La presencia de la tecnología digital en sí misma no es garantía de que sus beneficios particulares serán aplicados a favor del aprendizaje.

\section{Agradecimientos}

Proyecto PAPIIT IN404818 "Entornos híbridos de Aprendizaje diseño de problemas prototípicos en contextos reales y digitales".

\section{Declaración de conflicto de intereses}

Los autores declaran no tener conflicto de intereses con respecto a la investigación, autoría o publicación de este artículo.

\section{Referencias}

Behm Rosas, H. (1969). Proyectos y realizaciones en América Latina. Aspectos del planeamiento físico de las universidades. CONESCAL, 12, 1071-1077.

Budka, P. (2011). From cyber to digital anthropology to an anthropology of the contemporary. Recuperado de http:// www.media-anthropology.net/file/budka_contemporary.pdf

Clark, A. y Chalmers, D. J. (2011). La mente extendida. CIC Cuadernos de Información y Comunicación, 16. https://doi. org/10.5209/rev_CIYC.2011.v16.1

Clark, J. E. (2010). The Digital Imperative: Making the Case for a 21st-Century Pedagogy. Computers and Composition, 27(1), 27-35. https://doi.org/10.1016/j.compcom.2009.12.004

Davisson, A. y Leone, A. C. (2017). From Coercion to Community Building. Technological Affordances as Rhetorical Forms. En Theorizing Digital Rhetoric (pp. 85-97). Routledge. https://doi.org/https://doi. org/10.4324/9781315203645

Fuentes Hernández, P. (2007). Campus universitarios en Chile: nuevas formas análogas a la ciudad tradicional. Atenea (Concepción), (496), 117-144. https://doi.org/10.4067/S0718-04622007000200008

García, I., Peña-López, I., Johnson, L., Smith, R., Levine, A., \& Haywood, K. (2010). Informe Horizon: Edición Iberoamericana 2010. Austin, Texas: The New Media Consortium.

Gere, C. (2009). Digital culture. London: Reaktion Books.

Gibson, J. J. (1979). The Ecological Approach to Visual Perception. Psychology Press.

Lévy, P. (2007). Cibercultura. La cultura en la sociedad digital. Anthropos.

Lupion Torres, P. y Rama, C. (2010). La Educación Superior a Distancia en América Latina y el Caribe. Editora Unisul.

Maher, M. Lou, Skow, B. y Cicognani, A. (1999). Designing the virtual campus. Design Studies, 20(4), 319-342. https://doi.org/10.1016/S0142-694X(98)00043-X

Marín Díaz, V. y Romero López, M. A. (2009). La formación docente universitaria a través de las TICs. Pixel-Bit. Revista de Medios y Educación, 35, 97-103.

Meso Ayerdi, K., Pérez Dasilva, J. Á. y Mendiguren Galdospin, T. (2011). La implementación de las redes sociales en la enseñanza superior universitaria. Tejuelo, 12(1), 137-155. Recuperado de http://hdl.handle.net/10662/4555

Rickards, J. (2000). The Virtual Campus: the impact on teaching and learning. En Proceedings of the IATUL Conferences. Recuperado de https://docs.lib.purdue.edu/cgi/viewcontent.cgi?article=1513\&context=iatul

Rueda, R. (2006). Tecnologías y escuela. En La razón técnica desafía a la razón escolar (pp. 27-42). Noveduc, Buenos Aires, Argentina. 
Sánchez Sordo, J. M. (2019). Cognición extendida en ambientes digitales de aprendizaje. Universidad Nacional Autónoma de México. Recuperado de http://132.248.9.195/ptd2019/enero/0784916/Index.html

Touriñán López, J. M., Olveira Olveira, M. E. y Rodríguez Martínez, A. (2003). La sociedad de la información y las nuevas exigencias en la formación del profesorado. Aula Abierta, (81), 57-74. Recuperado de https:// dialnet.unirioja.es/servlet/articulo? codigo $=756028$

Universidad Nacional Autónoma de México. (2009). Estatuto del Sistema Universidad Abierta y Educación a Distancia. En Gaceta UNAM.

Urbina, S. y Salinas, J. (2014). Campus virtuales: una perspectiva evolutiva y tendencias. Revista De Educación a Distancia, (42). Recuperado de https://revistas.um.es/red/article/view/236601 